\title{
Research of ACO in Decision-Making of Skills and Tactics in Badminton
}

\author{
Jie Mao \\ College of Sports Engineering and Information Technology \\ Wuhan Sports University \\ Wuhan, China \\ e-mail: jiem027@163.com
}

\begin{abstract}
This study focuses on ant colony optimization (ACO) in decision making of skills and tactics in badminton. The model about decision making of skills and tactics in badminton based on ACO is put forward and explained, with the ultimate objective to win in competitive sports relying on skills and tactics. According to the technical and tactical characteristics of badminton competition concluded based on item group theory, the simulation model of ant colony system is formulated, providing theoretical support for the algorithm design, and vividly explaining the principle and mechanism of ant colony's route search.
\end{abstract}

Keywords-ACO;badminton; decision-making.

\section{INTRODUCTION}

Nowadays, the swarm intelligence theory and application algorithm have been gradually applied in the research of optimization in many fields, followed by unexpected achievement. According to a variety of researches, swarm intelligence show sound search effect in both discrete solution space and continuous solution space, and they can effectively solve combinatorial optimization related problems. ACO is not the best method to solve the traveling salesman problems, but it can provide new idea for solving combinatorial optimization related problems and quickly realize optimization. It is often typically applied to network routing optimization and data mining. Ant Colony Routing (ACR) firstly put forward by HP company and British Telecom in 1990s have been applied into many classical combinatorial optimization related case studies, such as weapon target assignment and optimization, vehicle running path planning, quadratic programming problem (QPP), robot path planning, process planning, graph coloring research, etc.

Table tennis, badminton, volleyball and tennis games are advantageous and potential advantageous events for Chinese players in athletics sports, and lots of theoretical studies have focused on the skills and tactics used in these sports. Of all the winning factors in the sports of the same item group, each contains definite technical and tactical meaning, while, in athletics sports, skills and tactics are critical factors for players to win net antagonistic events. To accurately and flexibly use and change the skills and tactics and make corresponding right decisions in the games is regarded as complex combinational optimization related problem, with characteristics of non-linearness, multi constraint and discreteness. In this study, the model about decision making of optimized winning skills and tactics in badminton based on ACO is put forward and explained, according to the technical and tactical characteristics of net-separated antagonistic sports, which means to provide reference for the application of technical and tactical decisions in these item groups.

\section{RESEARCH METHOD}

\section{A. Research object}

In this study, badminton as the research object.

\section{B. Research method}

- Method of literature review: The literature and monographs about theories of swarm intelligence and item group training are reviewed, and then the application of swarm intelligence theory is researched in depth.

- Theoretical analysis method: The theories of swarm intelligence and item group training are analyzed in depth.

- Numerical simulation method: The application of swarm intelligence - ACO in technical and tactical decision making in badminton is reasoned.

- Experimental research method: The model of optimal combination algorithm of skills and tactics used in badminton competition is proposed according to swarm intelligence theory or ACO.

\section{Technical route}

As is shown in Figure 1, this study contains the following steps, proposal of research question, determining of research content, selection of research method, analysis of ACO, data collection and analysis about skills and tactics used in badminton competition, design of study process with ACO, verification of ACO application according to the expectation. 


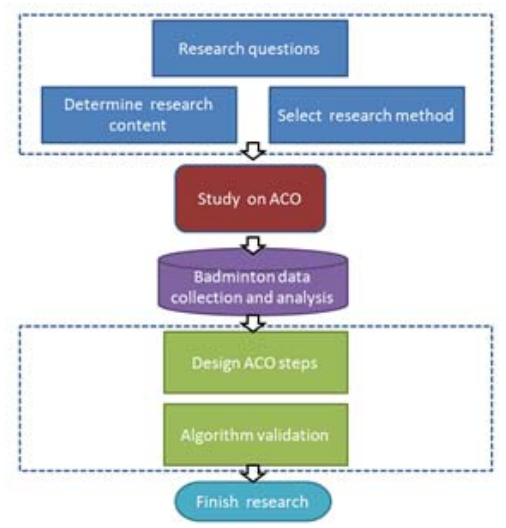

Figure 1. Technical route

\section{RESEARCH CONTENT}

\section{A. Principle of ACO}

ACO, a new optimization algorithm based on ant colony proposed in 1990s by M. Dorigo, an Italian scholar inspired by ant colony's specific actions, solves the well-known traveling salesman problem (TSP). The individual ant's behavior is simple, but the ant colony's behavior is complex. When foraging, ants can secrete certain chemical substance named as pheromone which then forms pheromone route that can transfer information for ant colony so as to complete complex tasks. In the above process, the information exchange and cooperation are critical for individuals.

In the movement, ant colony will leave pheromone substances in the ways which they must pass by so as to guide their movement direction, and they tend to the direction with higher pheromone intensity, which shows positive feedback, namely, the routes with more ant individuals are more likely to be preferred by the followers an to become optimal ones. In other words, ACO integrated with distributed computing, positive feedback mechanism and greedy search enjoys strong ability to search better solution. Positive feedback can quickly find better solutions, distributed computing stops premature convergence, and greedy search helps to find acceptable solution in the search process as early as possible, reducing the search duration.

\section{B. Theory of skills and tactics in badminton competition}

Of all athletics sports, badminton competition is advantageous for Chinese players, and lots of researches have focused on technical and tactical theory.

As is demonstrated by Tian Maijiu's study of item group theory, fast action, hardness, completeness, accuracy, flexibility are the winning factors in badminton competition (Table 1). In the same item group, the characteristics of skills and tactics are inevitable correlated. Skill and tactics decision-making ability is regarded as one of the important factors for winning badminton competition, and in the whole process, those players who can timely and unexpectedly change decisions of skills and tactics can control and finally win the whole game. Its common winning factors with other items contain fast action, flexibility, completeness, accuracy and hardness demonstrated by skills and tactics.

TABLE I. TABLE TyPE StYLES

\begin{tabular}{|c|c|}
\hline Item & Winning factor \\
\hline Badminton & fast action, flexibility, completeness, accuracy and hardness \\
\hline \multicolumn{2}{|c|}{ (Source: Tian Maijiu, "Item group training theory", 1998) }
\end{tabular}

The tactical quality and quantity in badminton competition mutually influence each other. The flexibly varying quantity can help to improve the tactical quality, while high-quality tactics can make up the insufficient tactical quantity.

\section{RESULT}

\section{A. Simulation model of ant colony system}

In the competition process, the combination of strategies is determined based on the skills and tactics frequently used by the two sides, their characteristics, the two sides' response and resistance ability. According to the technical and tactical characteristics in confrontation, the combination of our stronger confrontation ability and quicker response as well as the other side's slower response and weaker confrontation ability is more likely to lead to our winning; the most ideal route optimization combination of the whole competition process does not mean the most ideal optimization in each confrontation; therefore, it is necessary to design different routes of confrontation combinations according to the opponent's status. It is supposed that, in the confrontation process, there are two combinations of skills and tactics for our side's winning, $\mathrm{AC}$ and $\mathrm{BD}$. The combination $\mathrm{AB}$ is our side's confrontation strategy, while the combination CD is the other side's coping strategy (Figure 2), wherein numbers refer to comprehensive index of confrontation capacity and time for response, and the smallest number means optimization.

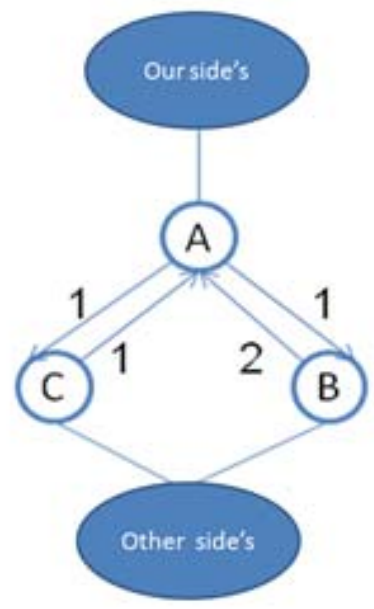

Figure 2. Simulation model of ant colony system

Simulation process of ACO is explained below: 
When $t=0$, the possibility for the other side to move toward $\mathrm{B}$ is the same as that toward $\mathrm{C}$, respectively for route $\mathrm{AB}$ or $\mathrm{AC}$

According to our side's judgment, the comprehensive index of the other side's confrontational strategy is CA $=1$ and $\mathrm{BA}=2$; the comprehensive index of our selection of $\mathrm{AB}$ and $\mathrm{AC}$ line is both 1 , with $\mathrm{AB}$ rather than $\mathrm{AC}$ for confrontation, and in next confrontation process, our side may encounter the same combination of tactics and skills and thus can refer to route $\mathrm{AB}$ for confrontation. The technical and tactical characteristics of the same item group are inevitably correlated, so the model proposed in this study can be applied to the analysis of winning skills and tactics used in all items of net-separated antagonistic item group.

This is just an ideal theoretical confrontation model, while, in practice, comprehensive index of confrontation strategy should be also considered due to the athletes' different ability of perception and response, which indicates the difficulties of theoretical study of competitive sports items based on ACO.

\section{B. ACO model}

Based on the above description of the characteristic of ACO, corresponding ACO about technical and tactical decisions in badminton competition is established for skill and tactics optimization in reality. The technical and tactical decision optimization based on ACO is explained below:

1) Given that iteration number is I which is initialized as 0 , the pheromone $\tau$ ij on various technical and tactical decision routes are initialized;

2) $\mathrm{m}$ ants are randomly placed at the terminal of technical and tactical decision routes, and then the technical and tactical decision tabu list is respectively designed for our side and the other side;

3) The route for optimal technical and tactical decision-making is determined according to the above technical and tactical decision tabu list.

4) Solution: Each ant can work out solution according to the rule of state change and form technical and tactical decision route. The ant is burdened with the task to find the optimal route in line with the following formula.

Wherein $\tau$ ij represents our side's technical and tactical decision route, $\eta \mathrm{ij}$ refers to the other side's technical and tactical decision route, allowed $\mathrm{k}=(0,1, \cdots, \mathrm{n}-1)$ indicates the next point of attack allowed for the ant k. $\alpha$ and $\beta$ are respectively the information accumulated during the ant's movement and the parameter of the importance of route selection according to the heuristic information. In the formula, a tabu list tabu $\mathrm{k}(\mathrm{k}=1,2, \ldots, \mathrm{m})$ is established for each ant has, indicating the process the ant $\mathrm{k}$ has completed at time t. This ant is not allowed to repeat the process in this cycle, and after this cycle, the tabu list is cleared.

5) The information of each confrontation process is recorded, including that of time and skills and tactics combination. The data in tabu table are updated, and accordingly the realization of the optimal winning strategy is determined, otherwise, go to step 4) to repeat the process for optimal solution, and then continue step 6);
6) If the optimal winning strategy based on technical and tactical decision is realized, the confrontation process is completed. If the number of iterations is equal to Imax, the process is ended, and optimal solution is selected from all of the feasible solutions; otherwise, $\mathrm{I}=\mathrm{I}+1$, go to step 2) for the next evolution.

The algorithm flow is shown as follows:

$$
p_{i j}^{k} \begin{cases}\frac{\tau_{i j}^{\alpha}(t) \cdot \eta_{i j}^{\beta}(t)}{\sum_{s \in a l l o w e d} \tau_{k}^{\alpha}(t) \cdot \eta_{i s}^{\beta}(t)}, & \text { if } j \in \text { allowed }_{k} \\ 0 \quad, & \text { if not }\end{cases}
$$

\section{CONCLUSIONS AND SUGGESTIONS}

\section{A. Conclusions}

In this study, the simulation model of ant colony system in confrontation process of badminton competition is designed by referring to the ant colony optimization theory and the literature about theories of skill and tactics used in badminton competition, and accordingly the optimal algorithm model for wining skill and tactic decision-making in badminton competition is established, which lays theoretical foundation for the research of application of confrontation decision optimization in badminton competition, provides mathematical reference for in-depth study of winning factors in item group, and analysis, and provides reference for the analysis of skills and tactics in badminton competition.

\section{B. Suggestions}

Due to the special characteristics of competitive sports, the ACO should be researched with the method different from that for other fields. According to item group theory, the same item group has both its generality and different features, and therefore, it is necessary to explore the essence and connotation of the items in depth, without over overgeneralization.

\section{ACKNOWLEDGMENT}

We thank Lu Qi and Du Yunyun for helpful discussions and the referees for greatly improving the manuscript. The research was supported by scientific research fund of Huibei provincial education department (item number: Q20134101).

\section{REFERENCES}

[1] Zhang jun. Ant Colony Optimization[M].Beijing:Tsinghua University Press, 2007.1

[2] Shifeng,Wanghui,Yulei,Hufei.Analysis of Matlab intelligent algorithm in 30 cases[M]. Beijing: Beihang University Press, 2011.7

[3] Tian maijiu.Item group training theory[M].Beijing: People's Sports Press, 1998.6

[4] Tian maijiu. Sports training[M].Beijing: China Higher Education Press,2006

[5] Mao jie. Ant Colony Optimization in Skill and Tactic Decision of Competitive Sport[J]. Journal of Sports Adult Education,2012(4):4344

[6] Wang lingxia,Zhao hong,Wang wanjun.Hybrid ant colony optimization to solve the distributed system and task allocation 
problem[J].Automation and Instruments,2014(12):178-179,183 Automation and Instruments Automation and Instruments Automation and InstrumentsAutomation and InstrumentsAutomation and Instruments

[7] Wang qiming,Li zhanguo,Fan aiyuan.Quantum ant colony algorithm based on game theory[J]. Journal of Shandong university(engineering science) .2015(2):1-5

[8] Xia lirong,etc. An adaptive multi-objective particle swarm optimization algorithm based on dynamic AHP and its application[J]. Control and Decision,2015(2):215-221

[9] Zhao kai,Li benwei,Li haining. Comprehensive evaluation of aeroengine performance bansed on improved $\mathrm{PSO}[\mathrm{J}]$.Aeroengine, 2014(6):13-17
[10] Xia yamei,etc. Optimizing services composition based on improved Ant colony Algorithm[J].Chinese Journal of Computers,2012(2):270281

[11] Liu zheng. Research and Simulation of Blast Furnace System based on ACO[J]. Automation Applications,2014(12):1-2

[12] Zhao yaguang. Research on the algorithm of WSN data fusion based on ant colony algorithm and BP neural network[D].Yunan Unveirsity,2013

[13] Li feng.Ant Clustering Energy Cluster in 802.11 Wireless Network Localization[J].Computer and Digital Engineering,2014(12):22472250 